Article original

\title{
Données préliminaires sur l'écologie des chauves-souris frugivores de la commune du Plateau (Abidjan, Côte d'Ivoire)
}

\author{
Coffi J. M. NiAmien*, Hilaire K. YaOKoKoré-BéıBro, Inza KonÉ \& Kouakou E. N'Goran
}

Laboratoire de Zoologie et de Biologie Animale, UFR Biosciences, Université de Cocody-Abidjan, Côte d'Ivoire, 22 BP 582 Abidjan 22 *Auteur pour les Correspondances (E-mail :niamiencoffi@yahoo.fr)

Reçu le 04-06-09, accepté le 04-02-2010.

\begin{abstract}
Résumé
La communauté de chauves-souris frugivores de la Commune du Plateau à Abidjan a été étudiée d'août 2005 à juillet 2006. Cette étude a été réalisée en procédant à des observations directes et des comptages aux pieds d'arbres en vue de connaître les espèces présentes, leur effectif et d'établir leur préférence d'habitat. Les résultats indiquent que cette commune héberge deux espèces de Mégachiroptères, la chauve-souris paillée, Eidolon helvum (Kerr, 1792) représentée par une très forte colonie et la chauve-souris à tête de marteau, Hypsignathus monstrosus $\mathrm{H}$. Allen, 1861, observée pour la première fois et représentée par quelques individus. L'effectif de la population de chauves-souris paillées est en moyenne de $185049 \pm 1291,5$ individus. Les effectifs ont varié selon le site et la saison. Les chauves-souris paillées ont une préférence d'habitat marquée pour les sites dortoirs à Mangifera indica L. (Anacardiaceae).
\end{abstract}

Mots clés : Chauves-souris frugivores, inventaire, habitat, milieu urbain.

\begin{abstract}
Primary data on the ecology of fruit bats in Plateau council (Abidjan, Côte d'Ivoire)

The bat community of the Plateau Council in Abidjan has been studied from August 2005 through July 2006. Direct observations and roost counts were used in order to identify the number of species, the population size and identify their preferred roosting trees. Results show that the Plateau bat community is composed of two species of fruit bats, the largely abundant straw-colored fruit bats, Eidolon helvum (Kerr, 1792) and the rare hammer-headed bat, Hypsignathus monstrosus $\mathrm{H}$. Allen, 1861 observed for the first time. The population size of the straw-colored fruit bats was $185049 \pm 1291,5$ individuals during the study period. There was a spatial and seasonal variation of population size. The straw-colored fruit bats of Plateau demonstrated a high preference for roosting sites dominated by mangoe trees, Mangifera indica L. (Anacardiaceae).
\end{abstract}

Keys words : Fruit bats, inventory, habitat, urban area.

\section{Introduction}

L'ordre des Chiroptères présente des intérêts d'ordre systématique (Farh \& Ebigbo, 2004), écologique (Taylor et al., 2000 ; Chatelain et al., 2001), pharmacologique (Gonin, 2000), vétérinaire (Raharimanga et al., 2003), médical (Monath, 1999) et économique (Taylor et al., 2000). En dépit de ces multiples intérêts, peu de données écologiques les concernant sont disponibles en raison de leurs activités crépusculaires et surtout nocturnes (Thomas, 1982 ; Conservation International, 2001).

En Côte d'Ivoire, la ville d'Abidjan, et notamment la commune du Plateau abrite une importante communauté de chauves-souris frugivores, très peu étudiée (Huggel-wolf \& Huggel-wolf, 1965 ; Thomas, 1983, Niamien, 2004). Or, ces animaux contribuent à la pollinisation des fleurs et à la reconstitution des écosystèmes forestiers à travers la dispersion des graines des fruits consommés 
(Thomas, 1991 ; Taylor et al., 2000). En outre, ce sont les seuls animaux capables d'assurer la dispersion et favoriser la germination des graines d'Iroko (Milicia excelsa Welw. (Moraceae) et Chlorophora excelsa (Welw.) Benth. (Moraceae)), menacées par l'exploitation forestière en Afrique de l'Ouest (Hawthorme, 1995; Taylor et al., 2000). Ainsi, la commune du Plateau, de par la présence d'une importante communauté de chauves-souris frugivores devrait être considérée comme un site stratégiquement important pour la conservation de la biodiversité. L'importance de cette commune se situe également dans le fait qu'elle soit utilisée saisonnièrement par des colonies de chauvessouris paillées migratrices reproductrices comme site de maternité et de nurseries (Thomas, 1982, 1983 ; Niamien, 2004).

Cependant, il n'est pas rare d'y observer des abattages d'arbres utilisés par ces chauves-souris comme dortoirs (Niamien, 2004). Ceci constitue une menace sérieuse pour la communauté de chauves-souris du Plateau. En effet, ces organismes ont des besoins d'habitats spécifiques dont la destruction conduit à la réduction des effectifs d'animaux et à leur disparition (Myers, 1980 ; Fenton \& Thomas, 1985 ; Thomas, 1988 ; Barnes, 1990 ; Stone, et al., 1991 ; Skole \& Tucker, 1993 ; Farh \& Ebigbo, 2004).

La présente étude vise à connaître la composition spécifique de la communauté de chauves-souris frugivores de la commune du Plateau, son effectif et le mode d'utilisation des habitats.

\section{Matériel et méthodes}

\subsection{Site d'étude}

La ville d'Abidjan se situe dans le Sud de la Côte d'Ivoire. Elle comprend plusieurs communes dont celle du Plateau, qui s'étend entre $4^{\circ} 10^{\prime}$ et $4^{\circ} 50^{\prime}$ de longitudes Ouest et $5^{\circ} 10^{\prime}$ et $5^{\circ} 80^{\prime}$ de latitudes Nord, sur une superficie de $2,5 \mathrm{~km}^{2}$. Cette commune abrite dans sa partie centrale, une importante communauté de chauves-souris frugivores utilisant comme dortoir les différentes espèces d'arbres jonchant ses rues et avenues.

Le climat de la ville d'Abidjan et celui de la zone sud du pays, est caractérisé par quatre saisons : la petite saison sèche (d'août à septembre), la petite saison de pluies (d'octobre à novembre), la grande saison sèche (de décembre à mars) et la grande saison de pluies (d'avril à juillet) (Brou, 1997).

\subsection{Collecte des données}

L'inventaire des espèces de chauves-souris frugivores de la commune du Plateau a été effectué de façon pédestre, d'août 2005 à juillet 2006, dans sa partie centrale (zone d'étude) sur une surface de $0,028 \mathrm{~km}^{2}$ (Figure 1). La surface prospectée est caractérisée par une forte concentration d'arbres où la présence de chauves-souris frugivores est régulièrement observée. Les visites ont été réalisées les samedis, entre 7 heures et 17 heures pour tenir compte des activités de braconnage qui s'y déroulent les autres jours non ouvrables. Les observations ont été faites de façon directe avec une paire de jumelles et l'identification des espèces présentes à l'aide des guides d'identification de Kingdon (1997) et de Nowak (1999). Par ailleurs, les espèces d'arbres ont été identifiées avec l'aide du Centre National de Floristique de l'Université de Cocody. Au total 16 sites d'une superficie globale de $0,006 \mathrm{~km}^{2}$, essentiellement exploités comme dortoir ont été déterminés.

Pour déterminer les effectifs de chauves-souris de la commune du plateau, quatre comptages mensuels ont été réalisés. Ces comptages ont été réalisés aux pieds d'arbres, une fois par semaine, dans tous les sites dortoirs. Les fréquences d'utilisation des espèces d'arbres dortoirs exploitées par les chauves-souris ont été également déterminées. Ces dénombrements ont été faits par temps clair, les samedis, à l'exception des jours de matchs de football au Stade Felix Houphouët Boigny, contiguë à la zone d'étude. En effet, pendant les matchs de football, les cris des spectateurs entraînent des envolées massives de chauves-souris. Ces comptages ont été faits entre 7 heures et 17 heures avant toute activité nocturne des chauves-souris. Les comptages sont faits en dénombrant les chauves-souris perchées sur toutes les branches de chaque arbre dortoir, de la base des branches vers les extrémités sans distinction de sexe et d'âge, à l'aide d'une paire de jumelles et d'un compteur manuel (Thomas \& LaVal, 1987 ; Wiles et al., 1989). Les coordonnées géographiques des sites dortoirs ont été relevées à l'aide d'un «Global Position System» (G.P.S) de marque MAGELLAN. 


\subsection{Analyses statistiques}

Pour comparer les effectifs de chauves-souris paillées par site et par espèce d'arbre nous avons procédé à une analyse de variances après avoir vérifié la normalité des données avec le test de Shapiro-Wilk. Les fréquences d'utilisation des espèces d'arbres ont été comparées en utilisant le test de Khi-deux. A la suite de ces analyses, pour déterminer le site et l'espèce d'arbre dortoir abritant les plus grands effectifs de chauvessouris paillées, le test Post Hoc de comparaison et de classification de Newman-Keuls au seuil de $5 \%$ a été utilisé. Nous avons en outre testé les effets nombre d'espèces d'arbres par site et mois sur la distribution des chauves-souris paillées en utilisant le modèle linéaire généralisé. Tous les tests statistiques ont été effectués avec le logiciel STATISTICA (version 6.0).

\section{Résultats}

\subsection{Inventaire floristique des sites dortoirs}

Les 16 sites dortoirs (Figure 1) sont peuplés de 251 pieds d'arbres appartenant à quatre espèces : 147 pieds $(53,65 \%)$ de Samanea saman Merr. (Mimosaceae), 46 pieds $(17,15 \%)$ de Terminalia catappa L. (Combretaceae), 45 pieds $(16,42 \%)$ de Hevea brasiliensis Kunt. (Euphorbiaceae) et 36 pieds $(12,78 \%)$ de Mangifera indica L. (Anacardiaceae). Le nombre d'arbres par site varie de 6 à 34. Le peuplement floristique est dominé par $S$. saman, à l'exception des sites 2,3 et 4 où $H$. brasiliensis est abondant ; des sites 13 et 14 où $T$. catappa est dominant alors que sur les sites 9 et $12 \mathrm{M}$. indica est prépondérant (Tableau 1).

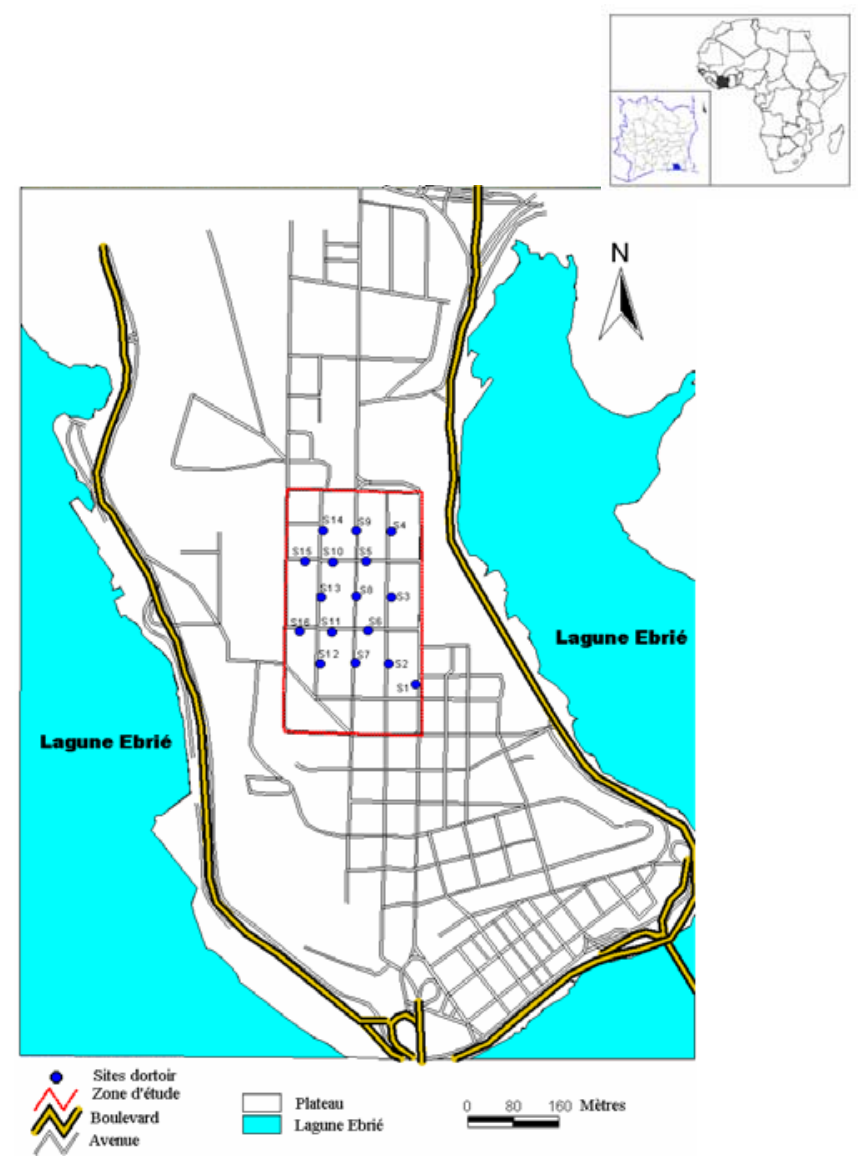

Figure 1 : Cartographie des sites dortoirs (numérotés de 1 à 16) des chauves-souris paillées de la commune du Plateau à Abidjan d'août 2005 à juillet 2006. 
Tableau 1: Résultats des inventaires floristiques des 16 sites dortoirs des chauves-souris paillées d'août 2005 à juillet 2006 dans la commune du Plateau d'Abidjan.

\begin{tabular}{|c|c|c|c|c|c|c|c|}
\hline $\begin{array}{l}\text { Sites } \\
\text { dortoirs }\end{array}$ & $\begin{array}{c}\text { Samanea } \\
\text { saman }\end{array}$ & $\begin{array}{c}\text { Terminalia } \\
\text { Catappa }\end{array}$ & $\begin{array}{c}\text { Hevea } \\
\text { brasiliensis }\end{array}$ & $\begin{array}{c}\text { Mangifera } \\
\text { indica }\end{array}$ & Total & $\begin{array}{c}\text { Surface } \\
\qquad\left(\mathrm{m}^{2}\right)\end{array}$ & $\begin{array}{l}\text { Densités } \\
\left(\text { Pied } / \mathrm{m}^{2}\right)\end{array}$ \\
\hline Site 1 & 7 & 0 & 0 & 1 & 8 & 324 & 0,03 \\
\hline Site 2 & 10 & 0 & 11 & 0 & 21 & 594 & 0,04 \\
\hline Site 3 & 12 & 0 & 16 & 0 & 28 & 594 & 0,05 \\
\hline Site 4 & 9 & 0 & 16 & 0 & 25 & 594 & 0,04 \\
\hline Site 5 & 8 & 0 & 0 & 0 & 8 & 162 & 0,05 \\
\hline Site 6 & 9 & 0 & 0 & 0 & 9 & 162 & 0,05 \\
\hline Site 7 & 5 & 0 & 0 & 1 & 6 & 594 & 0,01 \\
\hline Site 8 & 28 & 0 & 0 & 6 & 34 & 594 & 0,06 \\
\hline Site 9 & 8 & 2 & 0 & 15 & 25 & 594 & 0,04 \\
\hline Site 10 & 8 & 0 & 0 & 0 & 8 & 162 & 0,05 \\
\hline Site 11 & 13 & 0 & 0 & 0 & 13 & 162 & 0,08 \\
\hline Site 12 & 0 & 9 & 1 & 10 & 20 & 594 & 0,03 \\
\hline Site 13 & 0 & 22 & 0 & 0 & 22 & 594 & 0,04 \\
\hline Site 14 & 0 & 13 & 1 & 2 & 16 & 324 & 0,05 \\
\hline Site 15 & 13 & 0 & 0 & 1 & 14 & 162 & 0,08 \\
\hline Site 16 & 17 & 0 & 0 & 0 & 17 & 162 & 0,1 \\
\hline Total & 147 & 46 & 45 & 36 & 274 & 6372 & \\
\hline Proportions (\%) & 53,65 & 17,15 & 16,42 & 12,78 & 100 & & \\
\hline
\end{tabular}

\subsection{Inventaire qualitatif et quantitatif des chauves-souris}

Les inventaires qualitatifs ont permis d'identifier deux espèces de Mégachiroptères, la chauvesouris paillée, Eidolon helvum (Kerr, 1792) (Figure 2), qui est de loin l'espèce la plus fréquente et la chauve-souris à tête de marteau, Hypsignathus monstrosus $\mathrm{H}$. Allen, 1861 (Figure 3).

Les effectifs moyens mensuels de chauves-souris paillées par site sont consignés dans le Tableau 2. Au total, 2220584 chauves-souris ont été dénombrées. L'effectif moyen mensuel de la commune du Plateau est de $185049 \pm 1291,5$ individus. Les effectifs mensuels ont augmenté très significativement d'août à novembre ( $\mathrm{ddl}=3$; $F=31,15 ; p<0,001)$, se sont stabilisés jusqu'en février (ddl $=3 ; F=2,1 ; p>0,05$ ) avant de chuter progressivement et de façon sensible de mars à juillet (ddl $=4 ; F=15 ; p<0,05)$. Le plus grand effectif de chauves-souris paillées a été observé en février (grande saison sèche) alors que le plus petit effectif a été observé en juillet (grande saison de pluies) (Tableau 2).

Un effectif cumulé de 290 mâles de chauvessouris à tête de marteau a été dénombré sur les sept mois où l'espèce a été observée (août, septembre, octobre, février, mars, avril et mai), après la dispersion des chauves-souris paillées, lorsque les mâles perchés sur chaque pied d'arbre ont émis des appels sexuels au sein du seul site 13 (Figure 1).

Le modèle linéaire généralisé révèle qu'à l'exception du site (GLM : ddl = $15 ; W=12,56 ; p$ > $0,05)$, les paramètres que sont l'espèce d'arbre (GLM : ddl $=3 ; W=58,38 ; p<0,001$ ), le nombre d'espèce d'arbre par site (GLM : ddl $=3 ; \mathrm{W}=58,38$; $p<0,05)$ et l'association site-espèce d'arbre (GLM : ddl = $45 ; W=191,36 ; p<0,001$ ) influencent significativement la distribution des chauves-souris. 


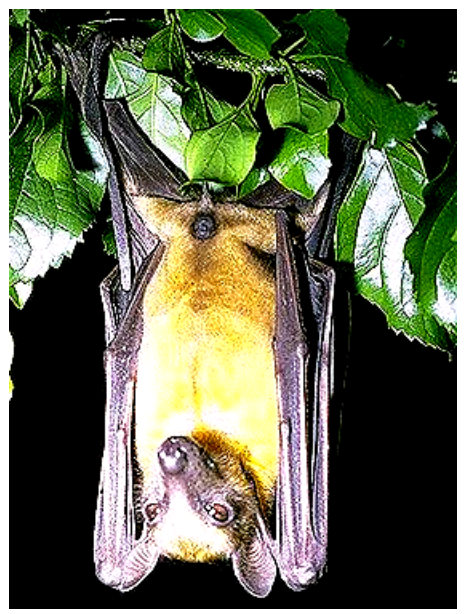

Figure 2 : Spécimen femelle de Eidolon helvum (Source : Université de Lubee, 2005).

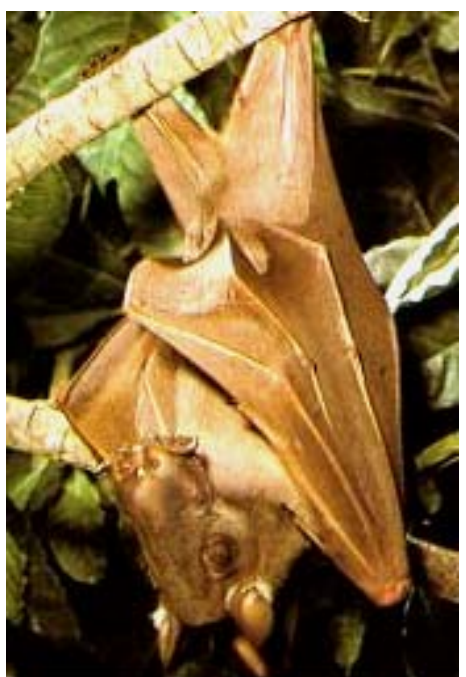

Figure 3 : Spécimen mâle de Hypsignathus monstrosus (Source : Université de Lubee, 2005).

\subsection{Variations spatiales et temporelles des effectifs de chauves-souris paillées}

Globalement, les effectifs moyens de chauvessouris paillées varient d'un site à l'autre $(\mathrm{ddl}=$ $15 ; F=7,62 ; p<0,001)$. Le test Post Hoc de Newman-Keuls révèle que les effectifs les plus élevés ont été obtenus aux sites 3, 5, 8 et 9 (Tableau 2).

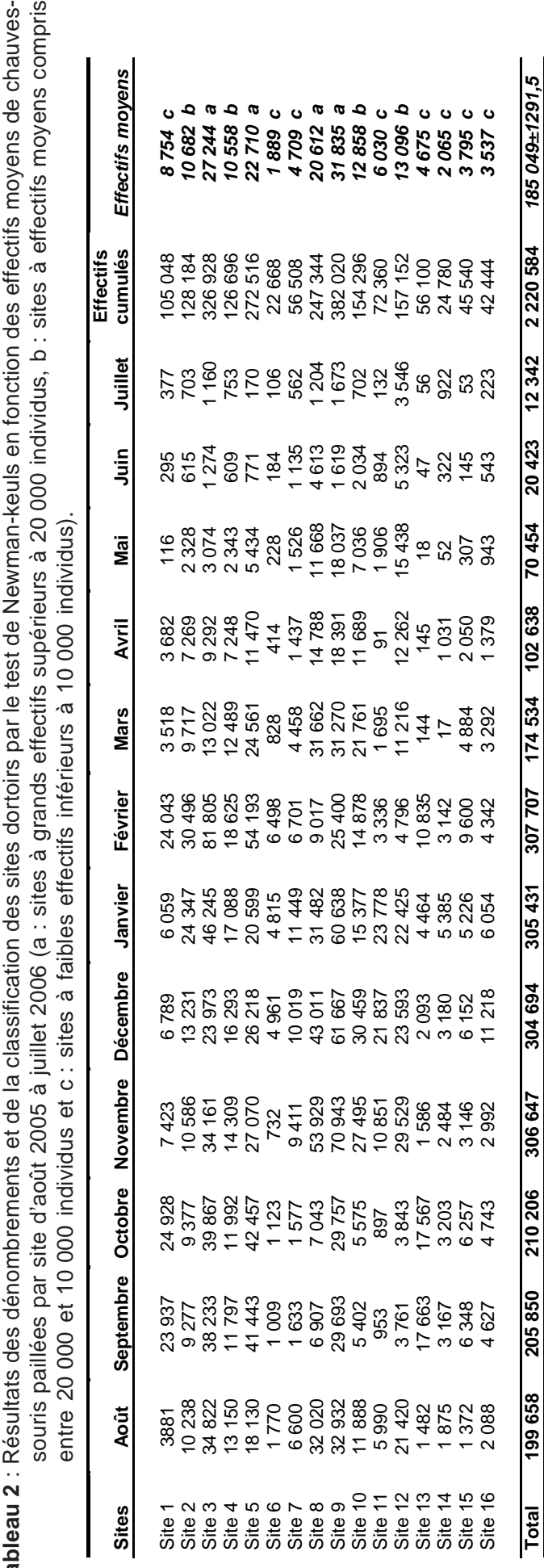


Au niveau mensuel, les sites dortoirs 3 et 5 présentent les plus grands effectifs de chauvessouris paillées pendant les mois d'août et septembre (petite saison sèche). Au cours des mois d'octobre et de novembre (petite saison de pluies), ce sont les sites 5 et 9 qui sont les plus exploités tandis que de décembre à mars (grande saison sèche), il s'agit de trois sites: site 3 (février), site 8 (mars) et du site 9 (décembre à janvier et d'avril à mai). En revanche, en juin et juillet (grande saison de pluies), le site 12 est le plus occupé (Tableau 2).

Le modèle linéaire généralisé confirme ces observations en relevant que le site (GLM : ddl = $15 ; W=81,7 ; p<0,001)$ et le mois (GLM : ddl = $3 ; W=11,45 ; p<0,05)$ ont significativement influencé la distribution des chauves-souris paillées au cours de l'étude $(p<0,05)$.

L'analyse de variances révèle que les effectifs de chauves-souris paillées varient très significativement en fonction de l'espèce d'arbre $(\mathrm{ddl}=3 ; \mathrm{F}=17,59 ; p<0,001)$. Le test de comparaison Post Hoc de Newman-Keuls montre que Mangifera indica a concentré les grands effectifs de chauves-souris paillées, Samanea saman les effectifs intermédiaires, Hevea brasiliensis et Terminalia catappa les plus faibles effectifs. En outre, les effectifs observés sur ces deux dernières espèces d'arbres ne diffèrent pas significativement selon le test de Newman-Keuls (Tableau 3).

Les effectifs moyens mensuels de chauvessouris paillées par espèce d'arbre dortoir montrent que quelle que soit la période, $M$. indica reste l'espèce d'arbre la plus prisée (Tableau 3 ) et que le mois (GLM : ddl =11; W = 21,7; $p<$ 0,001 ) et l'espèce d'arbre (GLM : ddl $=3 ; \mathrm{W}=$ 14,$17 ; p<0,001$ ) influencent la distribution des chauves-souris paillées.

Les fréquences d'exploitation des pieds d'arbres utilisés par les chauves-souris paillées varient très significativement avec l'espèce $\left(\chi^{2}=\right.$ $16,92 ; p<0,001)$. La comparaison des fréquences d'utilisation des espèces par le test de Khi-deux révèle que $H$. brasiliensis et $M$. indica ont été les espèces les plus fréquemment exploitées par les chauves-souris paillées. S. saman a été moyennement utilisée tandis que $T$. catappa est l'espèce la moins colonisée (Tableau 3).

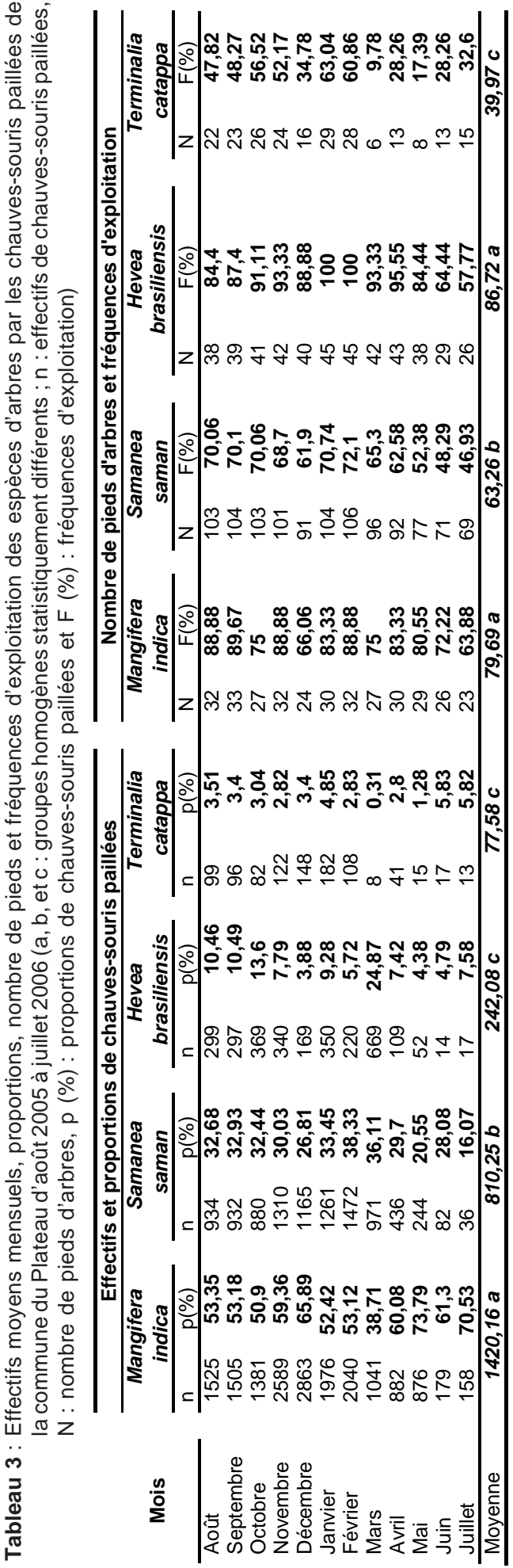


Les fréquences d'utilisation mensuelle moyenne des espèces d'arbres par les chauves-souris paillées font apparaître que $M$. indica a été plus exploitée d'août à septembre (petite saison sèche) et de juin à juillet (grande saison de pluies) tandis que $H$. brasiliensis l'a été au cours des autres mois (petite saison de pluies, grande saison sèche et grande saison de pluies) (Tableau 3). La distribution des chauves-souris paillées par pied d'arbre a varié significativement avec l'espèce (GLM : ddl $=3 ; W=25,22 ; p<0,001$ ) et le mois (GLM : ddl $=11 ; W=36,15 ; p<0,001$ ).

\section{Discussion}

Cette étude, signale pour la première fois dans la commune du Plateau la présence de la chauvesouris à tête de marteau, Hypsignathus monstrosus en plus de l'espèce Eidolon helvum identifiée auparavant (Huggel-wolf \& Huggel-wolf, 1965 ; Thomas, 1982, 1983). En outre, le comportement sexuel affiché, consistant à l'émission d'appels sexuels caractéristiques «Kwok» nous conforte dans cette identification (Bradbury, 1977 ; Thomas, 1982 ; Koné, 1996 ; Kingdon, 1997 ; Nowak, 1999).

L'alimentation semble être la cause de variation des effectifs de chauves-souris (Thomas, 1982, 1983 ; Dumont, 2003 ; Richter \& Cumming, 2006). Ainsi, l'augmentation des effectifs de chauves-souris paillées (d'août à novembre) serait en rapport avec la disponibilité des ressources alimentaires. En effet, la hausse des effectifs est le fait des colonies de reproduction en provenance de la savane (réserve de Lamto), qui en raison de la raréfaction locale de nourriture, migrent vers la commune du Plateau pour exploiter la diversité et l'abondance de nourriture compte tenu de leurs besoins énergétiques élevés (Thomas, 1982, 1983 ; De Frees \& Wilson, 1988 ; Cosson et al., 1996 ; Dumont, 2003 ; Nelson, 2003). Par la suite, la limitation de la nourriture assurerait le maintien des effectifs (novembre à février). En effet, chez les Mammifères, la taille du groupe est limitée par la disponibilité de la nourriture, vu que le succès reproductif des femelles est fonction de la quantité d'énergie acquise par l'alimentation (Janson, 1992 ; Cumming \& Bernard, 1997 ; Fleming \& Eby, 2003). Enfin, dès que les ressources alimentaires s'amenuisent ou sont épuisées, les individus reproducteurs retournent vers les savanes pour tirer profit de l'abondance de la production fruitière, d'où la baisse des effectifs (mars à juillet) (Van Schaik et al., 1993 ; Hepburn \& Radloff, 1995 ; Fleming \& Eby, 2003).

Le choix des sites semble être dicté par des considérations alimentaires. En effet, le regroupement des chauves-souris paillées sur $M$. indica a été plus marqué bien que cet arbre ne soit pas le plus abondant dans la zone d'étude et présente moins de branches étalées comparativement à S. saman dont l'architecture offre plus de possibilité de perchage à un plus grand nombre de chauves-souris paillées. Cela traduit ainsi, la préférence des chauves-souris paillées pour cette espèce d'arbre (Thomas, 1982). La présence de mangues mûres d'avril à juin (grande saison de pluies) au sein des sites dortoirs mentionnés ou à proximité, semble être un facteur déterminant dans le choix des habitats par ces animaux frugivores (Snow, 1971 ; Gautier-Hion, 1990 ; Erikson \& Ehrlen, 1991). La colonisation des sites à $M$. indica aurait ainsi un triple avantage car, ces sites leur serviraient à la fois de dortoir, de site d'alimentation et de nurseries. Ce choix contribuerait à réduire les coûts énergétiques liés à la locomotion (Aronson \& Givnish, 1983 ; Richter \& Cumming, 2006) et augmenterait de ce fait, leur succès reproductif (Wrangham, 1980 ; Terborgh \& Janson, 1986 ; Dunbar, 1988 ; Janson, 1992). Outre le facteur alimentaire, la préférence pour certains sites pourrait être liée à des aspects sécuritaires. En effet, les chauves-souris paillées de la commune du Plateau font l'objet de chasse par les riverains, qui se servent de lance-pierres pour les abattre (Niamien, 2004). Cela pourrait expliquer le fait qu'on les trouve le plus souvent dans les zones où la pression de braconnage est la plus faible. En effet, elles affichent une nette préférence pour les alentours de la Direction de la Police Judiciaire (Sites 5 et 9), de l'Assemblée Nationale (Site 3) et de certains domiciles privés (Site 8), où une dissuasion semble être exercée sur les braconniers par la présence policière et militaire en faction. L'utilisation de $\mathrm{H}$. brasiliensis comme espèce d'arbre dortoir semble être également liée à l'activité de braconnage. En effet, en réponse à la pression de cette pratique, les chauvessouris paillées chercheraient à se mettre à l'abri (Tidemann \& Flavel, 1987). Le feuillage touffu de cette espèce d'arbre, offrirait une mauvaise visibilité aux braconniers, qui chercheraient à les 
localiser (Lima \& Dill, 1990 ; Koné, 2004), car pour un animal, la meilleure manière d'échapper à un prédateur humain est de se cacher (Koné, 2004). Cette étude suggère donc que la disponibilité de nourriture, les impératifs de la reproduction et la pression de braconnage interfèrent et guident le choix d'habitats de la population de chauves-souris paillées de la commune du Plateau à Abidjan.

\section{Conclusion}

II ressort de cette étude que deux espèces de Mégachiroptères, sont présentes dans la commune du Plateau, la chauve-souris paillée, Eidolon helvum et la chauve-souris à tête de marteau Hypsignathus monstrosus observée pour la première fois. Un total de $185049 \pm 1291,5$ chauves-souris paillées a été dénombré. La population a augmenté d'août à novembre, a été stable jusqu'en février avant de chuter de mars à juillet. Sur les 16 sites dortoirs, quatre (sites 3, 5, 8 et 9) présentent les meilleures conditions de sécurité et d'alimentation pour cette population. Quatre espèces d'arbres constituent les supports dortoirs. Parmi elles, le manguier (Mangifera indica) est l'espèce d'arbre la plus utilisée par cette population de chauves-souris paillées. Pour assurer l'intégrité de la communauté de chauves-souris frugivores, les sites 3 et 9 ainsi que les sites à $M$. indica (sites 8 et 12) sont à protéger car ils ont abrité les plus grands effectifs pendant la période de reproduction (de novembre à février) où des femelles portant leurs petits sont observées. En outre, des abattages sélectifs des espèces d'arbres épargnant à la fois $M$. indica et $S$. saman devraient être envisagées, vu que cette dernière, de par sa grande distribution, permet d'absorber de grands effectifs de chauves-souris paillées au cours des migrations saisonnières de colonies reproductrices. Par ailleurs, le site 13, site de reproduction de la chauve-souris à tête de marteau doit être maintenu intact à cause de sa sensibilité aux modifications de son environnement, susceptibles d'affecter son succès reproductif et partant sa survie.

\section{Remerciements}

Nous remercions le Centre National de Floristique de l'Université de Cocody pour l'identification des espèces d'arbres de la zone d'étude. Nos remerciements s'étendent à la Direction de la Protection de la Nature, au Service Technique de la municipalité du Plateau et à la Direction de la Police Judiciaire, qui nous ont délivré les autorisations nécessaires pour mener cette investigation. Enfin, nous voudrions exprimer notre reconnaissance au Professeur Thomas Donalson de l'Université de Sherbrook (Canada), qui nous a fait parvenir la bibliographie nécessaire à la rédaction de cet article.

\section{Références citées}

Aronson R.B., \& Givnish J.J., 1983. Optimal central-place foragers: A comparison with null hypotheses. Ecology 64: 395-399.

Barnes R.F.W., 1990. Deforestation trends in tropical Africa. Afr. J. Ecol. 28: 161-173.

Bradbury J.W., 1977. Lek mating behaviour in the hammer-headed-bat. Z. Tierpsychol. 45 : 225-245.

Brou Y., 1997. Analyse et dynamique de la pluviométrie en milieu forestier Ivoirien. Thèse de troisième cycle. Universté d'Abidjan, Cocody, Côte-d'Ivoire. 200 pp.

Chatelain C., Kadjo B., \& Koné I., 2001. Relation faune-flore dans le Parc National de Taï: Une étude bibliographique. Wageningen, Pays-Bas: Tropenbos-Côte d'Ivoire série 3. 166pp.

Conservation International 2001. De la forêt à la mer : Les liens de la biodiversité de la Guinée au Togo. Washington, USA: Conservation International. 78pp.

Cosson J.F., Tranier M., \& Colas F., 1996. On the occurrence and possible migratory behaviour of the fruit bat Eidolon helvum in Mauritania, Africa. J. Afr. Zool. 110: 369-371.

Cumming G.S., \& Bernard R.T.F., 1997. Rainfall, Food abundance and timing of parturition in African bats. Oecologia 111 : 309-317.

De frees S., \& Wilson D.E., 1988. Eidolon helvum. Mamm. Species 312 : 1-5.

Dumont E. R., 2003. Bat and fruit. In: Kunz T. \& Fenton M.B., Eds. Bat ecology. Chicago, USA : University of Chicago. pp. 398-429. 
Dunbar R.I.M., 1988. Evolution of the grouping patterns. In: Croom H., Eds. Primate social systems. London, England. pp. 106-150.

Eriksson O., \& Ehrlen J., 1991. Phenological variation in fruit characteristics in vertebrate dispersed plants. Oecologia 86 : 463-470.

Farh J., \& Ebigbo N., 2004. Evaluation rapide des Chiroptères dans la forêt classée du pic de Fon, Guinée. In : Conservation International. Une évaluation biologique rapide de la forêt classée du pic de fon, chaîne du Simandou, Guinée. Wasington, USA. pp. 171-180.

Fenton M.B., \& Thomas D.W., 1985. Migrations and dispersal of bats. In: Rankin M.A., Eds. Migration Mecchanisms and adaptative significance. New-york, USA. pp. 409-424.

Fleming T.H., \& Eby P., 2003. Ecology of bat migration. In: Kunz T.H. \& Fenton M.B., Eds. Bat ecology. Chicago, USA. pp. 156-208.

Gautier-Hion A., 1990. Coadaptations entre rythmes de fructification et frugivorie en forêt tropicale du Gabon: Mythe ou réalités. Rev. Ecol. 40 : 405-434.

Gonin X., 2000. Les chiroptères : Vie et mœurs. Génève, Suisse : Chiroptera. 66 pp.

Hawthorme W.D., 1995. Ecological profiles of Ghanian forest trees. Oxford, USA : Oxford forestry institute. $347 \mathrm{pp}$.

Hepburn H.R., \& Radloff S.E., 1995. First approximation to a phenology of the honeybees (Apis mellifera) and flora of Africa. Oecologia 101: 265-273.

Huggel-wolf H.J., \& Huggel-wolf M., 1965. La biologie de Eidolon helvum (Kerr) (Megachiroptera). Acta Trop. 22 : 1-10.

Janson C.A., 1992. Evolutionary ecology of primate groups. Ann. Rev. Ecol. Syst. 17: 95-130.

Kingdon J., 1997. Bats Chiroptera. In : Academic press., Eds. The Kingdon field guide to African Mammals. London, England. pp. 111-136.

Koné I., 1996. Le choix des sites d'appels sexuels par le mâle du Mégachiroptère Hypsignathus monstrosus H. Allen, 1861 dans le Parc National de Taï (Côte d'Ivoire). Mémoire de DEA. Université d'Abidjan, Cocody, Côte d'Ivoire. 36pp.
Koné I., 2004. Effet du braconnage sur quelques aspects du comportement du Colobe BaiProcolobus (Piliocolobus) badius (Kerr) et du Cercopithèque Diane-Cercopithecus diana diana (Linné) dans le parc national de Taï, Côte d'Ivoire. Wageningen, Pays-bas: Tropenbos Côte d'Ivoire, series 4. 112 pp.

Lima S.L., \& Dill L.M., 1990. Behavioural decisions made under the risk of predation: a review and prospectus. Can. J. Zool. 68: 619-640.

Monath T. P., 1999. Ecology of Marburg and Ebola virus : Speculation and directions for future research. J. Infect. Dis. 179 (1) : 127-138.

Myers N., 1980. Conversion of tropical moist forest. Washington, USA: National Academy of science. 205 pp.

Nelson S. L., 2003. Nutritional ecology of Old world fruit bats: a test of the calcium-constraint hypothesis. PhD. University of Florida, Gainesville, USA. 189 pp.

Niamien C.J.M., 2004. Dénombrement, activités spatio-temporelles et facteurs de menace de la population de chauves-souris paillées, Eidolon helvum (Kerr, 1792) de la Commune du Plateau à Abidjan (Côte d'Ivoire). Mémoire de DEA. Université d'Abidjan, Cocody, Côte d'Ivoire. 36pp.

Nowak R.M., 1999. Walker's Mammals of the world (Sixth edition, volume 2). Baltimore, USA Johns Hopkins University Press. 1248 pp.

Raharimanga V., Ariey F., Cardiff S.G., Goodman S.M., Tall A., Rousset D., \& Robert V., 2003. Haemoparasites of bats in Madagascar. Arch. Inst. Past. Madag. 69 (1-2) : 70-76.

Richter H.V., \& Cumming G.S., 2006. Food availability and annual migration of the strawcolored fruit bat (Eidolon helvum). J. Zool. 268 (1) : 35-44.

Skole D., \& Tucker C.J., 1993. Tropical deforestation and habitat fragmentation in the Amazon: Satellite data from 1978 to 1988. Sciences 260: $1905-1910$

Snow D.W., 1971. Evolutionary aspects of fruiteating by birds. Ibis 113: 194-202.

Stone T.A., Brown I.F., \& Woodwell G.M., 1991. Estimation by remote sensing of 
deforestation in central rondonia, Brazil. For. Ecol. Manag. 38: 291-304.

Taylor D.A.R., Kankan B.O., \& wagner M.R., 2000. The role of the bat fruit, Eidolon helvum in seed dispersal, survival, and germination in Milicia excela, a threatened West African hardwood. Biotropica 18 : 1-4.

Terborgh J., \& Janson C.H., 1986. The socioecology of primate groups. Annu. Rev. Ecol. Syst. 17 : 111-135.

Thomas D.W., 1982. The Ecology of an African savanna fruit bat community: Resource partitioning and role in seed dispersal. PhD. University of Aberdeen, Aberdeen, Scotland. 205pp.

Thomas D.W., 1983. The annual migration of three species of West African fruit bats (Chiroptera: Pteropodidae) Can. J. Zool. 61 : 2266-2272.

Thomas D.W., 1988. Analysis of diets of plantvisiting bats. In: Kunz T.H., Eds. Ecological and behavioral methods for the study of bats. Washington, USA : Smithsonian Institution Press. pp. 211-220.
Thomas D.W., 1991. On fruit, seed and bat. Biotropica 9: 8-13.

Thomas D.W., \& LaVal R.K., 1987. Survey and census methods. In : Kunz T.H., Eds. Ecological and Behavioral Methods for the study of Bats. Washington, USA : Smithsonian Institution Press. pp. 77-89.

Tidemann C.R., \& Flavel S.C., 1987. Factors affecting choice of diurnal roost site by treehole bats (Microchiroptera) in south-eastern Australia. Austr. Wildl. Res. 14 : 459-473.

Van Schaik P.C., Terborgh J.W., \& Wright S.J., 1993. The phenology of tropical forests: Adaptative significance and consequences for primary consumers. Annu. Rev. Ecol. Syst. 24: 353-377.

Wiles G.J., Lemke T.O., \& Payne N.H., 1989. Population estimates of fruit bats (Pteropus mariannus) in the Mariana Islands. Conserv. Biol. 3 : 66-76.

Wrangham R.W., 1980. An ecology model of female-bonded primate groups. Behaviour $75: 262-300$. 